\title{
A Biochemical study on the effect of some treatments on bone resorption and formation in osteoporotic rats
}

\author{
Naglaa Khedr ${ }^{1}$, Nahla El-Ashmawy ${ }^{1}$, Aly Hagagg ${ }^{1}$, Hoda El-Bahrawy ${ }^{1}$ and Eman El-Abd ${ }^{2}$ \\ ${ }^{1}$ Department of Biochemistry, Faculty of Pharmacy, Tanta University, Egypt \\ ${ }^{2}$ Medical Research Institute, Alexandria University, Egypt
}

\section{III}

Background: Osteoporosis thins bones, weakening them and making them more susceptible to fractures. It may be related to withdrawal of sex hormones by ageing. Aim: The present study was conducted to evaluate the bone protective effects of raloxifene, risedronate, and their combination on osteoporotic male and female rats. Materials and Methods: Osteoporosis was induced by orchidectomy (ORX) of 35 male Wistar rats and ovariectomy (OVX) of 50 female Wistar rats. Four weeks post-surgery, each of ORX and OVX rats were randomized into 4 groups: control, Raloxifene (RAL), Risedronate (RIS) and Raloxifene+Risedronate (RAL+RIS). RAL dose was $3 \mathrm{mg} / \mathrm{kg}$ given orally 3 times/week. RIS dose was $5 \mu \mathrm{g} / \mathrm{kg}$ given by s.c. injection, twice weekly. After 6 weeks of treatment, alkaline (ALP) and acid phosphatase (ACP) activities, bone mineral density (BMD), histochemical localization of ALP, gene expression of osteoprotegerin (OPG) and bone morphogenetic protein2(BMP-2) were examined. Results: The OVX and ORX rats showed a significant increase in plasma ALP and ACP activities and histochemical activity of ALP in ORX rats and OVX rats as compared with sham, and attenuated by treatment with RAL and RIS. BMD of femur didn't change after gonadectomy. In OVX-RAL and ORX-RIS, BMD was improved compared to untreated rats. Gene expression of OPG and BMP-2 was down regulated in OVX rats versus sham, but was up-regulated by RAL and RIS treatments. In ORX rats, OPG gene expression was up-regulated versus sham, but was down-regulated by RIS treatment. Conclusions: Both RAL and RIS reduced bone turnover and maintained BMD in osteoporotic rats.

Keywords: Osteoporosis; osteoprotegerin; Raloxifene; Risedronate

Editor-in-Chief: Prof. M.L. Salem, PhD - Article DOI: 10.21608/JCBR.2021.59559.1132 